\title{
Axiomatizations of the discrete Choquet integral and extensions
}

\author{
Miguel Couceiro Jean-Luc Marichal \\ Mathematics Research Unit, University of Luxembourg \\ 6, rue Coudenhove-Kalergi, L-1359 Luxembourg, Luxembourg \\ Email: \{miguel.couceiro, jean-luc.marichal\}@uni.lu
}

\begin{abstract}
Three important properties in aggregation theory are investigated, namely horizontal min-additivity, horizontal max-additivity, and comonotonic additivity, which are defined by certain relaxations of the Cauchy functional equation in several variables. We show that these properties are equivalent and we completely describe the functions characterized by them. By adding some regularity conditions, the latter functions coincide with the Lovász extensions vanishing at the origin, which subsume the discrete Choquet integrals. We also propose a simultaneous generalization of horizontal min-additivity and horizontal max-additivity, called horizontal medianadditivity, and we describe the corresponding function class. Additional conditions then reduce this class to that of symmetric Lovász extensions, which includes the discrete symmetric Choquet integrals.
\end{abstract}

Keywords: Aggregation function, discrete Choquet integral, discrete symmetric Choquet integral, Lovász extension, functional equation, Cauchy equation, comonotonic additivity, horizontal additivity.

\section{Introduction}

When we need to merge a set of numerical values into a single one, we usually make use of a so-called aggregation function, e.g., a mean or an averaging function. Various aggregation functions have been proposed thus far in the literature, thus giving rise to the growing theory of aggregation which proposes, analyzes, and characterizes aggregation function classes. For recent references, see Beliakov et al. [2] and Grabisch et al. [6].

A noteworthy aggregation function is the socalled (discrete) Choquet integral, which has been widely investigated in aggregation theory, due to its many applications for instance in decision making (see the edited book [7]). A convenient way to introduce the discrete Choquet integral is via the concept of Lovász extension. An $n$-place Lovász extension is a continuous function $f: \mathbb{R}^{n} \rightarrow \mathbb{R}$ whose restriction to each of the $n$ ! subdomains (induced by $\sigma \in S_{n}$ )

$\mathbb{R}_{\sigma}^{n}=\left\{\mathbf{x}=\left(x_{1}, \ldots, x_{n}\right) \in \mathbb{R}^{n}: x_{\sigma(1)} \leqslant \cdots \leqslant x_{\sigma(n)}\right\}$ is an affine function, where $S_{n}$ denotes the set of permutations on $[n]=\{1, \ldots, n\}$. An $n$-place Choquet integral is simply a nondecreasing (in each variable) $n$-place Lovász extension which vanishes at the origin. For general background, see [6, §5.4].

The class of $n$-place Choquet integrals has been axiomatized independently by means of two noteworthy aggregation properties, namely comonotonic additivity (see, e.g., [4]) and horizontal minadditivity (originally called "horizontal additivity", see $[3, \S 2.5])$. Recall that a function $f: \mathbb{R}^{n} \rightarrow \mathbb{R}$ is said to be comonotonically additive if, for every $\sigma \in S_{n}$, we have

$$
f\left(\mathbf{x}+\mathbf{x}^{\prime}\right)=f(\mathbf{x})+f\left(\mathbf{x}^{\prime}\right) \quad\left(\mathbf{x}, \mathbf{x}^{\prime} \in \mathbb{R}_{\sigma}^{n}\right) .
$$

To describe the second property, consider the horizontal min-additive decomposition of the $n$-tuple $\mathbf{x} \in \mathbb{R}^{n}$ obtained by "cutting" it with a real number $c$, namely

$$
\mathbf{x}=(\mathbf{x} \wedge c)+(\mathbf{x}-(\mathbf{x} \wedge c))
$$

where $\mathbf{x} \wedge c$ denotes the $n$-tuple whose $i$ th component is $x_{i} \wedge c=\min \left(x_{i}, c\right)$. A function $f: \mathbb{R}^{n} \rightarrow \mathbb{R}$ is said to be horizontally min-additive if

$$
f(\mathbf{x})=f(\mathbf{x} \wedge c)+f(\mathbf{x}-(\mathbf{x} \wedge c)) \quad\left(\mathbf{x} \in \mathbb{R}^{n}, c \in \mathbb{R}\right) .
$$

In this paper we completely describe the function classes axiomatized by each of these properties. More precisely, after recalling the definitions of Lovász extensions, discrete Choquet integrals, and their symmetric versions (Section 2), we show that comonotonic additivity and horizontal minadditivity (as well as its dual counterpart, namely horizontal max-additivity) are actually equivalent properties. We describe the function class axiomatized by these properties and we show that, up to certain regularity conditions (based on those we usually add to the Cauchy functional equation to get linear solutions only), these properties completely characterize those $n$-place Lovász extensions which vanish at the origin. Nondecreasing monotonicity is then added to characterize the class of $n$-place Choquet integrals (Section 3). We also introduce a weaker variant of the properties above, called horizontal median-additivity, and determine the function class axiomatized by this new property. Finally, by adding some natural properties, we 
characterize the class of $n$-place symmetric Lovász extensions and the subclass of $n$-place symmetric Choquet integrals.

We employ the following notation throughout the paper. Let $\mathbb{R}_{+}=\left[0, \infty\left[\right.\right.$ and $\left.\left.\mathbb{R}_{-}=\right]-\infty, 0\right]$. For every $A \subseteq[n]$, the symbol $\mathbf{1}_{A}$ denotes the $n$-tuple whose $i$ th component is 1 , if $i \in A$, and 0 , otherwise. Let also $\mathbf{1}=\mathbf{1}_{[n]}$ and $\mathbf{0}=\mathbf{1}_{\varnothing}$. The symbols $\wedge$ and $\vee$ denote the minimum and maximum functions, respectively. For every $\mathbf{x} \in \mathbb{R}^{n}$, let $\mathbf{x}^{+}=\mathbf{x} \vee 0$ and $\mathbf{x}^{-}=(-\mathbf{x})^{+}$. For every function $f: \mathbb{R}^{n} \rightarrow \mathbb{R}$, we define its diagonal section $\delta_{f}: \mathbb{R} \rightarrow \mathbb{R}$ by $\delta_{f}(x)=f(x \mathbf{1})$. More generally, for every $A \subseteq[n]$, we define the function $\delta_{f}^{A}: \mathbb{R} \rightarrow \mathbb{R}$ by $\delta_{f}^{A}(x)=f\left(x \mathbf{1}_{A}\right)$.

It is important to notice that comonotonic additivity as well as horizontal min-additivity and horizontal max-additivity extend the classical additivity property defined by the Cauchy functional equation for $n$-place functions

$$
f\left(\mathbf{x}+\mathbf{x}^{\prime}\right)=f(\mathbf{x})+f\left(\mathbf{x}^{\prime}\right) \quad\left(\mathbf{x}, \mathbf{x}^{\prime} \in \mathbb{R}^{n}\right) .
$$

In this regard, recall that the general solution $f: \mathbb{R}^{n} \rightarrow \mathbb{R}$ of the Cauchy equation (1) is given by $f(\mathbf{x})=\sum_{k=1}^{n} f_{k}\left(x_{k}\right)$, where the $f_{k}: \mathbb{R} \rightarrow \mathbb{R}$ $(k \in[n])$ are arbitrary solutions of the basic Cauchy equation $f_{k}\left(x+x^{\prime}\right)=f_{k}(x)+f_{k}\left(x^{\prime}\right)$ (see $\left.[1, \S 2-4]\right)$. As the following theorem states, under some regularity conditions, $f_{k}$ is necessarily a linear function.

Theorem 1 ([1]) Let $E=\mathbb{R}$ or $\mathbb{R}_{+}$or $\mathbb{R}_{-}$. If $f: E \rightarrow \mathbb{R}$ solves the basic Cauchy equation

$$
f\left(x+x^{\prime}\right)=f(x)+f\left(x^{\prime}\right)
$$

then either $f$ is of the form $f(x)=c x$ for some $c \in$ $\mathbb{R}$, or the graph of $f$ is everywhere dense in $\mathbb{R}^{2}$. The latter case is excluded as soon as $f$ is continuous at a point or monotonic or Lebesgue measurable or bounded from one side on a set of positive measure.

As we will see in this paper, comonotonic additivity, horizontal min-additivity, and horizontal median-additivity of a function $f: \mathbb{R}^{n} \rightarrow \mathbb{R}$ force the 1-place functions $\left.\delta_{f}^{A}\right|_{\mathbb{R}_{+}}$, and $\left.\delta_{f}^{A}\right|_{\mathbb{R}_{-}}(A \subseteq[n])$ to solve the basic Cauchy equation. Theorem 1 will hence be useful to describe the corresponding function classes whenever the regularity conditions stated are assumed.

Recall that a function $f: \mathbb{R}^{n} \rightarrow \mathbb{R}$ is said to be homogeneous (resp. positively homogeneous) of degree one if $f(c \mathbf{x})=c f(\mathbf{x})$ for every $\mathbf{x} \in \mathbb{R}^{n}$ and every $c \in \mathbb{R}$ (resp. every $c>0$ ).

\section{Lovász extensions and symmetric Lovász extensions}

We now recall the concept of Lovász extension and introduce that of symmetric Lovász extension.

Consider a pseudo-Boolean function, that is, a function $\phi:\{0,1\}^{n} \rightarrow \mathbb{R}$, and define the set function $v_{\phi}: 2^{[n]} \rightarrow \mathbb{R}$ by $v_{\phi}(A)=\phi\left(\mathbf{1}_{A}\right)$ for every $A \subseteq[n]$. Hammer and Rudeanu [8] showed that such a function has a unique representation as a multilinear polynomial of $n$ variables

$$
\phi(\mathbf{x})=\sum_{A \subseteq[n]} a_{\phi}(A) \prod_{i \in A} x_{i}
$$

where the set function $a_{\phi}: 2^{[n]} \rightarrow \mathbb{R}$, called the Möbius transform of $v_{\phi}$, is defined by

$$
a_{\phi}(A)=\sum_{B \subseteq A}(-1)^{|A|-|B|} v_{\phi}(B) .
$$

The Lovász extension of a pseudo-Boolean function $\phi:\{0,1\}^{n} \rightarrow \mathbb{R}$ is the function $f_{\phi}: \mathbb{R}^{n} \rightarrow \mathbb{R}$ whose restriction to each subdomain $\mathbb{R}_{\sigma}^{n}\left(\sigma \in S_{n}\right)$ is the unique affine function which agrees with $\phi$ at the $n+1$ vertices of the $n$-simplex $[0,1]^{n} \cap \mathbb{R}_{\sigma}^{n}$ (see $[9,11])$. We then have $\left.f_{\phi}\right|_{\{0,1\}^{n}}=\phi$.

It can be shown (see $[6, \S 5.4 .2]$ ) that the Lovász extension of a pseudo-Boolean function $\phi:\{0,1\}^{n} \rightarrow \mathbb{R}$ is the continuous function

$$
f_{\phi}(\mathbf{x})=\sum_{A \subseteq[n]} a_{\phi}(A) \bigwedge_{i \in A} x_{i} \quad\left(\mathbf{x} \in \mathbb{R}^{n}\right) .
$$

Its restriction to $\mathbb{R}_{\sigma}^{n}$ is the affine function

$$
\begin{aligned}
f_{\phi}(\mathbf{x})= & \phi(\mathbf{0})+x_{\sigma(1)} v_{\phi}\left(A_{\sigma}^{\uparrow}(1)\right) \\
& +\sum_{i=2}^{n}\left(x_{\sigma(i)}-x_{\sigma(i-1)}\right) v_{\phi}\left(A_{\sigma}^{\uparrow}(i)\right) \\
& -x_{\sigma(n)} \phi(\mathbf{0})
\end{aligned}
$$

for every $\mathbf{x} \in \mathbb{R}_{\sigma}^{n}$, and where $A_{\sigma}^{\uparrow}(i)=$ $\{\sigma(i), \ldots, \sigma(n)\}$. Indeed, both sides of (2) agree at $\mathbf{x}=\mathbf{0}$ and $\mathbf{x}=\mathbf{1}_{A_{\sigma}^{\uparrow}(k)}$ for every $k \in[n]$. Thus we see that $f_{\phi}-\phi(\mathbf{0})=f_{\phi-\phi(\mathbf{0})}$ is positively homogeneous of degree one.

We say that a function $f: \mathbb{R}^{n} \rightarrow \mathbb{R}$ is a Lovász extension if there is a pseudo-Boolean function $\phi:\{0,1\}^{n} \rightarrow \mathbb{R}$ such that $f=f_{\phi}$.

An $n$-place Choquet integral is a nondecreasing Lovász extension $f_{\phi}: \mathbb{R}^{n} \rightarrow \mathbb{R}$ such that $f_{\phi}(\mathbf{0})=0$. It is easy to see that a Lovász extension $f: \mathbb{R}^{n} \rightarrow$ $\mathbb{R}$ is an $n$-place Choquet integral if and only if its underlying pseudo-Boolean function $\phi=\left.f\right|_{\{0,1\}^{n}}$ is nondecreasing and vanishes at the origin (see $[6$, $\S 5.4])$.

We now introduce the concept of symmetric Lovász extension. Here "symmetric" does not refer to invariance under a permutation of variables but rather to the role of the origin of $\mathbb{R}^{n}$ as a symmetry center with respect to the function values whenever the function vanishes at the origin.

The symmetric Lovász extension of a pseudoBoolean function $\phi:\{0,1\}^{n} \rightarrow \mathbb{R}$ is the $n$-place function $\check{f}_{\phi}: \mathbb{R}^{n} \rightarrow \mathbb{R}$ defined by

$$
\check{f}_{\phi}(\mathbf{x})=f_{\phi}(\mathbf{0})+f_{\phi}\left(\mathbf{x}^{+}\right)-f_{\phi}\left(\mathbf{x}^{-}\right) \quad\left(\mathbf{x} \in \mathbb{R}^{n}\right),
$$


where $f_{\phi}$ is the Lovász extension of $\phi$. As immediate consequences of this definition, we see that $\check{f}_{\phi}$ is continuous and that

$$
\check{f}_{\phi-\phi(\mathbf{0})}(-\mathbf{x})=-\check{f}_{\phi-\phi(\mathbf{0})}(\mathbf{x}) \quad\left(\mathbf{x} \in \mathbb{R}^{n}\right) .
$$

More generally, we can easily see that $\check{f}_{\phi-\phi(\mathbf{0})}$ is homogeneous of degree one. Moreover, $\check{f}_{\phi}$ is nondecreasing if and only if so is $\phi$.

By (2) and (3), it follows that the restriction of $\check{f}_{\phi}$ to $\mathbb{R}_{\sigma}^{n}$ is the function

$$
\begin{aligned}
\check{f}_{\phi}(\mathbf{x}) & =\phi(\mathbf{0})+\left(-x_{\sigma(1)} \phi(\mathbf{0})\right. \\
& +\sum_{i=1}^{p-1}\left(x_{\sigma(i)}-x_{\sigma(i+1)}\right) v_{\phi}\left(A_{\sigma}^{\downarrow}(i)\right) \\
& \left.+x_{\sigma(p)} v_{\phi}\left(A_{\sigma}^{\downarrow}(p)\right)\right) \\
& +\left(x_{\sigma(p+1)} v_{\phi}\left(A_{\sigma}^{\uparrow}(p+1)\right)\right. \\
& +\sum_{i=p+2}^{n}\left(x_{\sigma(i)}-x_{\sigma(i-1)}\right) v_{\phi}\left(A_{\sigma}^{\uparrow}(i)\right) \\
& \left.-x_{\sigma(n)} \phi(\mathbf{0})\right) \quad\left(\mathbf{x} \in \mathbb{R}_{\sigma}^{n}\right)
\end{aligned}
$$

where $A_{\sigma}^{\uparrow}(i)=\{\sigma(i), \ldots, \sigma(n)\}, \quad A_{\sigma}^{\downarrow}(i)=$ $\{\sigma(1), \ldots, \sigma(i)\}$, and the integer $p \in\{0,1, \ldots, n\}$ is such that $x_{\sigma(p)}<0 \leqslant x_{\sigma(p+1)}$. In this formula the terms in the first (resp. second) parentheses are to be considered only if $p \geqslant 1$ (resp. $p \leqslant n-1$ ).

We say that a function $f: \mathbb{R}^{n} \rightarrow \mathbb{R}$ is a symmetric Lovász extension if there is a pseudo-Boolean function $\phi:\{0,1\}^{n} \rightarrow \mathbb{R}$ such that $f=\check{f}_{\phi}$.

Nondecreasing symmetric Lovász extensions vanishing at the origin, also called discrete symmetric Choquet integrals, were introduced by Šipoš [12] (see also $[6, \S 5.4])$.

\section{Axiomatizations of Lovász extensions}

In the present section we show that comonotonic additivity, horizontal min-additivity, and horizontal max-additivity are equivalent and we describe the corresponding function class. By adding certain regularity conditions, we then axiomatize the class of $n$-place Lovász extensions. We first recall the definitions of these properties.

Two $n$-tuples $\mathbf{x}, \mathbf{x}^{\prime} \in \mathbb{R}^{n}$ are said to be comonotonic if there exists $\sigma \in S_{n}$ such that $\mathbf{x}, \mathbf{x}^{\prime} \in \mathbb{R}_{\sigma}^{n}$. A function $f: \mathbb{R}^{n} \rightarrow \mathbb{R}$ is said to be comonotonically additive if, for every comonotonic $n$-tuples $\mathbf{x}, \mathbf{x}^{\prime} \in \mathbb{R}^{n}$, we have

$$
f\left(\mathbf{x}+\mathbf{x}^{\prime}\right)=f(\mathbf{x})+f\left(\mathbf{x}^{\prime}\right)
$$

Given $\mathbf{x} \in \mathbb{R}^{n}$ and $c \in \mathbb{R}$, let $\llbracket \mathbf{x} \rrbracket_{c}=\mathbf{x}-\mathbf{x} \wedge c$ and $\llbracket \mathbf{x} \rrbracket^{c}=\mathbf{x}-\mathbf{x} \vee c$. We say that a function $f: \mathbb{R}^{n} \rightarrow \mathbb{R}$ is
- horizontally min-additive if, for every $\mathbf{x} \in \mathbb{R}^{n}$ and every $c \in \mathbb{R}$, we have

$$
f(\mathbf{x})=f(\mathbf{x} \wedge c)+f\left(\llbracket \mathbf{x} \rrbracket_{c}\right) .
$$

- horizontally max-additive if, for every $\mathbf{x} \in \mathbb{R}^{n}$ and every $c \in \mathbb{R}$, we have

$$
f(\mathbf{x})=f(\mathbf{x} \vee c)+f\left(\llbracket \mathbf{x} \rrbracket^{c}\right) .
$$

We immediately observe that, since any $\mathbf{x} \in \mathbb{R}^{n}$ decomposes into the sum of the comonotonic $n$ tuples $\mathbf{x} \wedge c$ and $\llbracket \mathbf{x} \rrbracket_{c}$ for every $c \in \mathbb{R}$ (i.e., $\mathbf{x}=$ $\left.(\mathbf{x} \wedge c)+\llbracket \mathbf{x} \rrbracket_{c}\right)$, any comonotonically additive function is necessarily horizontally min-additive. Dually, any comonotonically additive function is horizontally max-additive.

We also observe that if $f: \mathbb{R}^{n} \rightarrow \mathbb{R}$ satisfies any of these properties, then necessarily $f(\mathbf{0})=0$ (just take $\mathbf{x}=\mathbf{x}^{\prime}=\mathbf{0}$ and $c=0$ in $\left.(5)-(7)\right)$.

Lemma 2 If $f: \mathbb{R}^{n} \rightarrow \mathbb{R}$ is horizontally minadditive (resp. horizontally max-additive) then $\delta_{f}^{A} \mid \mathbb{R}_{+}\left(\right.$resp. $\left.\left.\delta_{f}^{A}\right|_{\mathbb{R}_{-}}\right)$is additive for every $A \subseteq[n]$. Moreover, $\delta_{f}$ is additive and odd.

Remark 1 For a horizontally min-additive or horizontally max-additive function $f: \mathbb{R}^{n} \rightarrow \mathbb{R}$, the function $\delta_{f}^{A}$ need not be additive. For instance, consider the binary horizontally min-additive function $f\left(x_{1}, x_{2}\right)=x_{1} \wedge x_{2}$. For $x>0$ and $x^{\prime}=-x$, we have

$$
\delta_{f}^{\{1\}}(x-x)=0>-x=\delta_{f}^{\{1\}}(x)+\delta_{f}^{\{1\}}(-x) .
$$

Theorem 3 A function $f: \mathbb{R}^{n} \rightarrow \mathbb{R}$ is horizontally min-additive if and only if there exists $g: \mathbb{R}^{n} \rightarrow \mathbb{R}$, with $\delta_{g}$ and $\left.\delta_{g}^{A}\right|_{\mathbb{R}_{+}}$additive for every $A \subseteq[n]$, such that, for every $\sigma \in S_{n}$,

$$
f(\mathbf{x})=\delta_{g}\left(x_{\sigma(1)}\right)+\sum_{i=2}^{n} \delta_{g}^{A_{\sigma}^{\uparrow}(i)}\left(x_{\sigma(i)}-x_{\sigma(i-1)}\right),
$$

for every $\mathbf{x} \in \mathbb{R}_{\sigma}^{n}$. In this case, we can choose $g=f$.

Similarly, we obtain the following dual characterization.

Theorem 4 A function $f: \mathbb{R}^{n} \rightarrow \mathbb{R}$ is horizontally max-additive if and only if there exists $h: \mathbb{R}^{n} \rightarrow \mathbb{R}$, with $\delta_{h}$ and $\left.\delta_{h}^{A}\right|_{\mathbb{R}_{-}}$additive for every $A \subseteq[n]$, such that, for every $\sigma \in S_{n}$,

$$
f(\mathbf{x})=\delta_{h}\left(x_{\sigma(n)}\right)+\sum_{i=1}^{n-1} \delta_{h}^{A_{\sigma}^{\downarrow}(i)}\left(x_{\sigma(i)}-x_{\sigma(i+1)}\right),
$$

for every $\mathbf{x} \in \mathbb{R}_{\sigma}^{n}$. In this case, we can choose $h=f$.

Theorem 5 For any function $f: \mathbb{R}^{n} \rightarrow \mathbb{R}$, the following assertions are equivalent.

(i) $f$ is comonotonically additive.

(ii) $f$ is horizontally min-additive. 
(iii) $f$ is horizontally max-additive.

If any of these conditions is fulfilled, then $\delta_{f},\left.\delta_{f}^{A}\right|_{\mathbb{R}_{+}}$, and $\left.\delta_{f}^{A}\right|_{\mathbb{R}_{-}}$are additive for every $A \subseteq[n]$.

Remark 2 (a) Theorems 3 and 4 provide two equivalent representations of comonotonically additive functions (see Theorem 5). For instance, for a binary comonotonically additive function $f: \mathbb{R}^{2} \rightarrow \mathbb{R}$, we have the representations

$$
\begin{aligned}
& f\left(x_{1}, x_{2}\right)= \\
& \begin{cases}g\left(x_{1}, x_{1}\right)+g\left(0, x_{2}-x_{1}\right), & \text { if } x_{1} \leqslant x_{2}, \\
g\left(x_{2}, x_{2}\right)+g\left(x_{1}-x_{2}, 0\right), & \text { if } x_{1} \geqslant x_{2},\end{cases}
\end{aligned}
$$

and

$$
\begin{aligned}
& f\left(x_{1}, x_{2}\right)= \\
& \begin{cases}h\left(x_{2}, x_{2}\right)+h\left(x_{1}-x_{2}, 0\right), & \text { if } x_{1} \leqslant x_{2}, \\
h\left(x_{1}, x_{1}\right)+h\left(0, x_{2}-x_{1}\right), & \text { if } x_{1} \geqslant x_{2},\end{cases}
\end{aligned}
$$

where $\delta_{g},\left.\delta_{g}^{A}\right|_{\mathbb{R}_{+}}, \quad \delta_{h}$, and $\left.\delta_{h}^{A}\right|_{\mathbb{R}_{-}}$are additive for every $A \subseteq[2]$. Thus, $f$ is completely determined by its values on the $x_{1}$-axis, the $x_{2}$-axis, and the line $x_{2}=x_{1}$.

(b) More generally, every function $f: \mathbb{R}^{n} \rightarrow \mathbb{R}$ satisfying any of the conditions of Theorem 5 is completely determined by its values on the lines $\left\{x \mathbf{1}_{A}: x \in \mathbb{R}\right\}(A \subseteq[n])$.

We now axiomatize the class of $n$-place Lovász extensions.

Theorem 6 Let $f: \mathbb{R}^{n} \rightarrow \mathbb{R}$ be a function and let $f_{0}=f-f(\mathbf{0})$. Then $f$ is a Lovász extension if and only if the following conditions hold:

(i) $f_{0}$ is comonotonically additive or horizontally min-additive or horizontally max-additive.

(ii) Each of the maps $\delta_{f_{0}}$ and $\left.\delta_{f_{0}}^{A}\right|_{\mathbb{R}_{+}}(A \subseteq[n])$ is continuous at a point or monotonic or Lebesgue measurable or bounded from one side on a set of positive measure.

The set $\mathbb{R}_{+}$can be replaced by $\mathbb{R}_{-}$in $($ii $)$. Condition (ii) holds whenever Condition $(i)$ holds and $\delta_{f_{0}}^{A}$ is positively homogeneous of degree one for every $A \subseteq$ $[n]$.

Remark 3 (a) Since any Lovász extension vanishing at the origin is positively homogeneous of degree one, Condition (ii) of Theorem 6 can be replaced by the stronger condition: $f_{0}$ is positively homogeneous of degree one.

(b) Axiomatizations of the class of n-place Choquet integrals can be immediately derived from Theorem 6 by adding nondecreasing monotonicity. Similar axiomatizations using comonotone additivity (resp. horizontal min-additivity) were obtained by de Campos and Bolaños [4] (resp. by Benvenuti et al. [3, \$2.5]). (c) The concept of comonotonic additivity appeared first in Dellacherie [5] and then in Schmeidler [10]. The concept of horizontal minadditivity was previously considered by Šipoš [12] and then by Benvenuti et al. [3, \$2.3] where it was called "horizontal additivity".

\section{Axiomatizations of symmetric Lovász extensions}

In this final section we introduce a simultaneous generalization of horizontal min-additivity and horizontal max-additivity, called horizontal medianadditivity, and we describe the corresponding function class. By adding further conditions, we then axiomatize the class of $n$-place symmetric Lovász extensions.

Horizontal median-additivity in a sense combines horizontal min-additivity and horizontal maxadditivity by using two cut levels that are symmetric with respect to the origin. Formally, we say that a function $f: \mathbb{R}^{n} \rightarrow \mathbb{R}$ is horizontally medianadditive if, for every $\mathbf{x} \in \mathbb{R}^{n}$ and every $c \geqslant 0$, we have

$$
f(\mathbf{x})=f(\operatorname{med}(-c, \mathbf{x}, c))+f\left(\llbracket \mathbf{x} \rrbracket_{c}\right)+f\left(\llbracket \mathbf{x} \rrbracket^{-c}\right),
$$

where $\operatorname{med}(-c, \mathbf{x}, c)$ is the $n$-tuple whose $i$ th component is the middle value of $\left\{-c, x_{i}, c\right\}$.

Since any $\mathbf{x} \in \mathbb{R}^{n}$ decomposes into the sum of the comonotonic $n$-tuples $\operatorname{med}(-c, \mathbf{x}, c)+\llbracket \mathbf{x} \rrbracket^{-c}=$ $\mathbf{x} \wedge c$ and $\llbracket \mathbf{x} \rrbracket_{c}$ for every $c \geqslant 0$ (i.e., $\mathbf{x}=$ $\left.\operatorname{med}(-c, \mathbf{x}, c)+\llbracket \mathbf{x} \rrbracket_{c}+\llbracket \mathbf{x} \rrbracket^{-c}\right)$, any comonotonically additive function is necessarily horizontally medianadditive. However, we will see that the converse claim is not true.

We also observe that if $f: \mathbb{R}^{n} \rightarrow \mathbb{R}$ is horizontally median-additive, then necessarily $f(\mathbf{0})=0$ (take $\mathbf{x}=\mathbf{0}$ and $c=0$ in (9)). We then see that

$$
f(\mathbf{x})=f\left(\mathbf{x}^{+}\right)+f\left(-\mathbf{x}^{-}\right) \quad\left(\mathbf{x} \in \mathbb{R}^{n}\right)
$$

(take $c=0$ in $(9)$ ). This observation motivates the following definitions.

We say that a function $f: \mathbb{R}^{n} \rightarrow \mathbb{R}$ is

- positively comonotonically additive if (5) holds for every comonotonic $n$-tuples $\mathbf{x}, \mathbf{x}^{\prime} \geqslant \mathbf{0}$.

- negatively comonotonically additive if (5) holds for every comonotonic $n$-tuples $\mathbf{x}, \mathbf{x}^{\prime} \leqslant \mathbf{0}$.

- positively horizontally min-additive if (6) holds for every $\mathbf{x} \geqslant \mathbf{0}$ and every $c \geqslant 0$.

- negatively horizontally max-additive if (7) holds for every $\mathbf{x} \leqslant \mathbf{0}$ and every $c \leqslant 0$.

We observe that if $f: \mathbb{R}^{n} \rightarrow \mathbb{R}$ satisfies any of the four properties above, then $f(\mathbf{0})=0$.

Lemma 7 For any function $f: \mathbb{R}^{n} \rightarrow \mathbb{R}$, the following assertions are equivalent.

(i) $f$ is horizontally median-additive. 
(ii) $f$ is positively horizontally min-additive, negatively horizontally max-additive, and satisfies (10).

(iii) There exists a positively horizontally minadditive function $g: \mathbb{R}^{n} \rightarrow \mathbb{R}$ and a negatively horizontally max-additive function $h: \mathbb{R}^{n} \rightarrow \mathbb{R}$ such that $f(\mathbf{x})=g\left(\mathbf{x}^{+}\right)+h\left(-\mathbf{x}^{-}\right)$for every $\mathbf{x} \in \mathbb{R}^{n}$.

By Lemma 7, to describe the class of horizontally median-additive functions, it suffices to describe the class of positively horizontally min-additive functions and that of negatively horizontally maxadditive functions. These descriptions are given in the following two theorems.

Theorem 8 For any function $f: \mathbb{R}^{n} \rightarrow \mathbb{R}$, the following assertions are equivalent.

(i) $f$ is positively horizontally min-additive.

(ii) $f$ is positively comonotonically additive.

(iii) There exists $g: \mathbb{R}^{n} \rightarrow \mathbb{R}$, with $\left.\delta_{g}^{A}\right|_{\mathbb{R}_{+}}$additive for every $A \subseteq[n]$, such that, for every $\sigma \in S_{n}$,

$$
f(\mathbf{x})=\delta_{g}\left(x_{\sigma(1)}\right)+\sum_{i=2}^{n} \delta_{g}^{A_{\sigma}^{\uparrow}(i)}\left(x_{\sigma(i)}-x_{\sigma(i-1)}\right)
$$

for every $\mathbf{x} \in \mathbb{R}_{\sigma}^{n} \cap \mathbb{R}_{+}^{n}$. In this case, we can choose $g=f$.

Theorem 9 For any function $f: \mathbb{R}^{n} \rightarrow \mathbb{R}$, the following assertions are equivalent.

(i) $f$ is negatively horizontally max-additive.

(ii) $f$ is negatively comonotonically additive.

(iii) There exists $h: \mathbb{R}^{n} \rightarrow \mathbb{R}$, with $\left.\delta_{h}^{A}\right|_{\mathbb{R}_{-}}$additive for every $A \subseteq[n]$, such that, for every $\sigma \in S_{n}$,

$$
f(\mathbf{x})=\delta_{h}\left(x_{\sigma(n)}\right)+\sum_{i=1}^{n-1} \delta_{h}^{A_{\sigma}^{\downarrow}(i)}\left(x_{\sigma(i)}-x_{\sigma(i+1)}\right),
$$

for every $\mathbf{x} \in \mathbb{R}_{\sigma}^{n} \cap \mathbb{R}_{+}^{n}$. In this case, we can choose $h=f$.

The following description of the class of horizontally median-additive functions follows immediately from Lemma 7 and Theorems 8 and 9 .

Theorem 10 For any function $f: \mathbb{R}^{n} \rightarrow \mathbb{R}$, the following assertions are equivalent.

(i) $f$ is horizontally median-additive.

(ii) $f$ is positively horizontally min-additive (or positively comonotonically additive), negatively horizontally max additive (or negatively comonotonically additive), and satisfies (10).

(iii) There exists $g: \mathbb{R}^{n} \rightarrow \mathbb{R}$ and $h: \mathbb{R}^{n} \rightarrow \mathbb{R}$, with $\left.\delta_{g}^{A}\right|_{\mathbb{R}_{+}}$and $\left.\delta_{h}^{A}\right|_{\mathbb{R}_{-}}$additive for every $A \subseteq[n]$, such that, for every $\sigma \in S_{n}$,

$$
\begin{aligned}
f(\mathbf{x})= & \delta_{g}^{A_{\sigma}^{\uparrow}(p+1)}\left(x_{\sigma(p+1)}\right) \\
& +\sum_{i=p+2}^{n} \delta_{g}^{A_{\sigma}^{\uparrow}(i)}\left(x_{\sigma(i)}-x_{\sigma(i-1)}\right) \\
& +\delta_{h}^{A_{\sigma}^{\downarrow}(p)}\left(x_{\sigma(p)}\right) \\
& +\sum_{i=1}^{p-1} \delta_{h}^{A_{\sigma}^{\downarrow}(i)}\left(x_{\sigma(i)}-x_{\sigma(i+1)}\right),(11)
\end{aligned}
$$

for every $\mathbf{x} \in \mathbb{R}_{\sigma}^{n}$, and where $p \in\{0, \ldots, n\}$ is such that $x_{\sigma(p)}<0 \leqslant x_{\sigma(p+1)}$. In this case, we can choose $g=h=f$.

We now axiomatize the class of $n$-place symmetric Lovász extensions.

Theorem 11 Let $f: \mathbb{R}^{n} \rightarrow \mathbb{R}$ be a function and let $f_{0}=f-f(\mathbf{0})$. Then $f$ is a symmetric Lovász extension if and only if the following conditions hold:

(i) $f_{0}$ is horizontally median-additive.

(ii) Each of the maps $\delta_{f_{0}}^{A} \mid \mathbb{R}_{+}$and $\delta_{f_{0}}^{A}{\mid \mathbb{R}_{-}}_{-}(A \subseteq[n])$ is continuous at a point or monotonic or Lebesgue measurable or bounded from one side on a set of positive measure.

(iii) $\delta_{f_{0}}^{A}(-1)=-\delta_{f_{0}}^{A}(1)$ for every $A \subseteq[n]$.

Conditions (ii) and (iii) hold together if and only if $\delta_{f_{0}}^{A}$ is homogeneous of degree one for every $A \subseteq[n]$.

Remark 4 (a) Since any symmetric Lovász extension vanishing at the origin is homogeneous of degree one, Conditions (ii) and (iii) of Theorem 11 can be replaced by the stronger condition: $f_{0}$ is homogeneous of degree one.

(b) Axiomatizations of the class of n-place symmetric Choquet integrals can be immediately derived by adding nondecreasing monotonicity.

The following proposition gives a condition for a symmetric Lovász extension to be a Lovász extension. This clearly shows that horizontal medianadditivity does not imply comonotonic additivity.

Proposition 12 For every $\phi:\{0,1\}^{n} \rightarrow \mathbb{R}$, we have $\check{f}_{\phi}=f_{\phi}$ if and only if $f_{\phi-\phi(\mathbf{0})}(-\mathbf{x})=$ $-f_{\phi-\phi(\mathbf{0})}(\mathbf{x})$ for every $\mathbf{x} \in \mathbb{R}_{+}^{n}$ (or equivalently, for every $\left.\mathbf{x} \in \mathbb{R}_{-}^{n}\right)$.

\section{Acknowledgments}

This research is supported by the internal research project F1R-MTH-PUL-09MRDO of the University of Luxembourg.

\section{References}

[1] J. Aczél and J. Dhombres. Functional equations in several variables. Encyclopedia of Mathematics and its Applications 31. Cambridge University Press, Cambridge, UK, 1989. 
[2] G. Beliakov, A. Pradera, and T. Calvo. Aggregation Functions: A Guide for Practitioners. Studies in Fuziness and Soft Computing. Springer, Berlin, 2007.

[3] P. Benvenuti, R. Mesiar, and D. Vivona. Monotone set functions-based integrals. In Handbook of measure theory, Vol. II, pages 1329-1379. NorthHolland, Amsterdam, 2002.

[4] L. M. de Campos and M. J. Bolaños. Characterization and comparison of Sugeno and Choquet integrals. Fuzzy Sets and Systems, 52(1):61-67, 1992.

[5] C. Dellacherie. Quelques commentaires sur les prolongements de capacités. In Séminaire de Probabilités, V (Univ. Strasbourg, année universitaire 19691970), pages 77-81. Lecture Notes in Math., Vol. 191. Springer, Berlin, 1971.

[6] M. Grabisch, J.-L. Marichal, R. Mesiar, and E. Pap. Aggregation functions. Encyclopedia of Mathematics and its Applications 127. Cambridge University Press, Cambridge, UK, 2009.

[7] M. Grabisch, T. Murofushi, and M. Sugeno, editors. Fuzzy measures and integrals - Theory and applications, volume 40 of Studies in Fuzziness and Soft Computing. Physica-Verlag, Heidelberg, 2000.

[8] P. Hammer and S. Rudeanu. Boolean methods in operations research and related areas. BerlinHeidelberg-New York: Springer-Verlag, 1968.

[9] L. Lovász. Submodular functions and convexity. In Mathematical programming, $11^{\text {th }}$ int. Symp., Bonn 1982, 235-25\%. 1983.

[10] D. Schmeidler. Integral representation without additivity. Proc. Amer. Math. Soc., 97(2):255-261, 1986.

[11] I. Singer. Extensions of functions of 0-1 variables and applications to combinatorial optimization. $\mathrm{Nu}$ mer. Funct. Anal. Optimization, 7:23-62, 1984.

[12] J. Šipoš. Integral with respect to a pre-measure. Mathematica Slovaca, 29(2):141-155, 1979. 